\title{
Specific protein-protein binding in many-component mixtures of proteins
}

\author{
Richard P. Sear \\ Department of Physics, University of Surrey, Guildford, Surrey GU2 7XH, United Kingdom \\ r.sear@surrey.ac.uk
}

\begin{abstract}
Proteins must bind to specific other proteins in vivo in order to function. The proteins must bind only to one or a few other proteins of the of order a thousand proteins typically present in vivo. Using a simple model of a protein, specific binding in many component mixtures is studied. It is found to be a demanding function in the sense that it demands that the binding sites of the proteins be encoded by long sequences of elements, and the requirement for specific binding then strongly constrains these sequences. This is quantified by the capability of proteins of a given size (sequence length), which is the maximum number of specific-binding interactions possible in a mixture. This calculation of the maximum number possible is in the same spirit as the work of Shannon and others on the maximum rate of communication through noisy channels.
\end{abstract}




\section{Introduction}

Proteins must interact to function, and they must interact with very high specificity in the highly complex mixtures that lie inside cells. For example, the 3 proteins Cdk2, cyclin and PCNA bind together in the nucleus to form a complex, which then goes on to interact with high specificity with a few other proteins [1]. $\quad(\mathrm{PCNA}=$ Proliferating Cell Nuclear Antigen and $\mathrm{Cdk} 2=$ Cyclin dependent kinase; they are both involved in cell division but also in other processes.) Cdk2 must bind strongly to cyclin and to PCNA, i.e., the complex must have a large equilibrium constant or equivalently a small dissociation constant. But Cdk2 must not bind to the of order one thousand other proteins present i.e., the equilibrium constants with all these other proteins must be small. This highly specific and strong binding in a mixture of thousands of different molecules is a demanding requirement and here we will try to quantify how demanding it is for a very simple model of a protein. To do so, we will rely on the fact that specific binding in a mixture is analogous to communication through a noisy channel, which has been extensively studied, in particular by Shannon $[2,3]$ and the many who have built on his work. In communication through a noisy channel reliable communication requires that all possible messages be sufficiently distinct from each other even after distortion by the noise. In specific binding in a mixture, a protein binding site or patch on the surface of a protein must be sufficiently distinct from all other patches to allow one other patch to bind to it with an equilibrium constant much larger than that of any other interaction of the patch. The theory developed by Shannon has been enormously useful in understanding communication systems and building efficient ones. We hope that applying the theory to protein-protein interactions in cells will be similarly useful.

This analogy to communication has been used for the similar problem of binding between a protein and a specific DNA sequence, in particular by Schneider [4-7]. See also [8] for other related work. Similar approaches have also been used extensively, see the review [9], in studies of our immune system, see [10] for an introduction to our immune system. Studies of the immune system look at a large set of immunesystem proteins recognising a single foreign protein. By contrast, our situation, a model of the cytoplasm of a prokaryote or a compartment of a eukaryote, involves not a single protein binding to another but binding between a very large number of pairs of proteins. However, there are close analogies between the specific binding required inside all cells and that required of immune-system proteins, in particular in both cases proteins must avoid binding to the wrong protein $[9,11,12]$. Inside cells, this would be all proteins bar a protein's partner, whereas in the case of the immune system, its proteins must not bind to other human proteins.

Our example of Cdk2 needing to bind to PCNA and to cyclin, is the rule rather than the exception in eukaryote cells [10]. We merely chose a specific example to make the discussion more concrete. The cells rely totally on a complex network or web of many specific interactions. This large set of interactions is often called the interactome, by analogy to the use of the word genome to denote the set of genes of an organism. In the study of noisy channels, a fundamental quantity of interest is the channel capacity $[2,3,13]$ : the maximum number of bits of information that can be sent per second. Shannon derived his famous channel-capacity theorem $[2,3]$ which allows this channel capacity to be calculated. Here we will calculate a related quantity for proteins, which we will call the capability. It is the maximum number of 
specific interactions, the maximum size of the interactome.

Protein interactions need to be understood to understand how cells work, but also the specificity of interactions is crucial to drug development. For example, Colas et al. [14] used a combinatorial-library approach to obtain peptide aptamers that bind specifically to Cdk2. Peptide aptamers are proteins that consist of a basic globular protein to form a 'scaffold' plus a region where the amino acids are allowed to vary. A library of these is generated and the peptide aptamers whose variable regions bind most strongly to the selected protein, here $\mathrm{Cdk} 2$, are selected. To function effectively, the potential drug, here the peptide aptamer, must interact in a highly specific way. If the requirement is to interfere with Cdk2, then the drug should bind to Cdk2 but not to the other thousands of proteins present. It is not sufficient that it bind strongly to Cdk2 in a dilute solution in vitro.

In the next section we will specify our mixture that is a model for the cytoplasm. It is an $N$ component mixture with highly specific interactions between the components. Section 3 defines the model of a protein. It is a simple lattice model in which each model protein has 6 binding sites or surface patches that mediate the specific interactions. Each patch is specified by a sequence of elements, and the interactions of a pair of patches is determined by their sequences. The model is not new, it was introduced in [15], but there only solubility, not specific binding was considered. The capability, the maximum number of interactions of a given high specificity, is calculated in section 4 , as a function of the length of these sequences. The next section, section 5, contains some illustrative results. The final section is a conclusion. An appendix contains a discussion of Shannon's channel-capacity theorem and a brief analysis along the lines of Schneider $[4,6]$.

\section{Model of the cytoplasm of a cell}

Our picture of the mixture of proteins inside a cell is of a large number of proteins, interacting via interactions of high specificity [10]. Our model of this is a mixture with $N$ distinct patches on proteins engaging in $N / 2$ pairwise interactions of high specificity. These are not necessarily due to $N$ proteins. $N$ is an even number.

In practice, in a cell the pairwise binding interactions vary widely in strength, and some protein binding sites, which we model by patches, bind to more than one other protein. Our model protein can be used to deal with this variation but for simplicity we take all $N / 2$ interactions to be equivalent. Also, as the number of proteins increases individual proteins become more dilute, this will tend to require larger equilibrium constants for binding. We neglect this effect here.

So, in a mixture with $N$ binding patches, each odd numbered patch $i$ is required to bind with the $(i+1)$ th patch, with a large equilibrium constant $K_{b}$. Now, the $i$ th $(i$ odd $)$ and $(i+1)$ th proteins must bind only to each other, it must be specific, and so in addition to binding to each other we require that the equilibrium constant for binding of patch $i$ to any patch other than patch $i+1$ be less than $K_{s} \ll K_{b}$. Similarly, the equilibrium constant between patch $i+1$ and any protein other than protein $i$ must also be less than $K_{s}$. Thus if we denote the binding constant between proteins $i$ and $j$ by $K_{i j}$ we require

$$
\begin{aligned}
& K_{i j}>K_{b} \quad|i-j|=1 \quad \min \{i, j\} \quad \text { odd } \\
& K_{i j}<K_{s} \text { otherwise }
\end{aligned}
$$


Figure 1. Schematic representation of a model protein, with the 3 visible patches represented by 'barcodes': a sequence of stripes, light for hydrophilic and dark for hydrophobic. The model shown has $n_{E}=4$ elements of which 2 are hydrophobic (0) and 2 are hydrophilic (1) in each case. For example, the 'barcode' of the front patch is 0101.

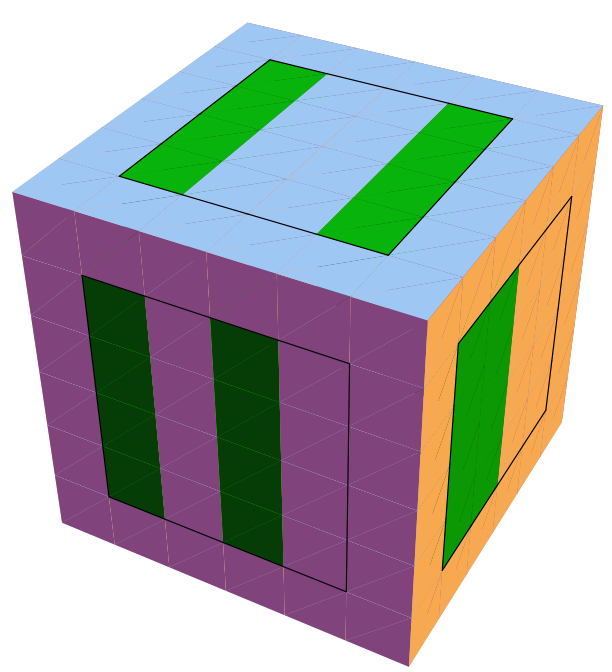

\section{Model protein}

Our model protein is as first defined in [15], although there only solubility, not binding was considered, and because of this only patches with rather short sequences were considered. The model protein is chosen to be as simple and as generic as possible, while having interactions which are mediated by surface patches whose interactions are a function of the values of a sequence or string of elements. Figure 1 is a schematic of the model. Note that although the elements determine the interactions in our model and the residues determine the interactions in a real protein, there is no one-to-one relationship between one of our elements and an amino acid.

The model protein is a cube, with each of its 6 faces having a single patch, for details see [15]. The lattice is cubic and each protein occupies 8 lattice sites arranged 2 by 2 by 2 . We make the model 2 sites across to reduce the range of the attraction, which is 1 site, to half the diameter of the hard core: see [16] where an earlier version of this model was defined and its behaviour compared to that of real proteins. The model proteins can rotate, and so have 24 distinct orientations. Each of the 6 faces of the cube has a patch. If the faces of 2 proteins are in contact there is an energy of interaction between the 2 touching patches of the 2 proteins. By in contact we mean that the faces must overlap completely otherwise the energy of interaction is taken to be zero. Also, the model is such that the energy of interaction between two touching patches is a constant which does not change when the two proteins are rotated about the axis joining their centres.

How a patch interacts is specified by a sequence or string of $n_{E}$ elements. If a element has a value of 
1 then the element is said to be hydrophilic or polar, whereas if it has a value of 0 then it is hydrophobic. A schematic of our model protein is shown in figure 1, where the sequence is represented by a 'barcode' with light stripes indicating $1 \mathrm{~s}$ and dark stripes indicating $0 \mathrm{~s}$. The interaction energy, $u_{i j}$, of a pair of touching patches, $i$ and $j$, is then given by

$$
u_{i j}=-\epsilon \sum_{\alpha=1}^{n_{E}}\left(b_{\alpha}^{(i)}-1\right)\left(b_{1+n_{E}-\alpha}^{(j)}-1\right),
$$

where $b_{\alpha}^{(i)}$ is element number $\alpha$ of patch $i . \epsilon$ is the interaction energy of 2 elements that are hydrophobic. We use energy units such that the thermal energy $k_{B} T=1$.

To calculate the interaction the string of elements of one of the patches is reversed and then the energy is just $\epsilon$ times the sum of the number of pairs of corresponding elements where both elements are 0 , are hydrophobic. The only interaction is between 2 hydrophobic elements; there is no hydrophobichydrophilic or hydrophilic-hydrophilic interaction. The reason one of the strings is reversed is that if this is not done then the interaction between like patches, $j=i$, is just $\epsilon$ times the number of $0 \mathrm{~s}$ in the string. Then all patches would stick to themselves. Reversing the strings removes this problem in a simple way. Of course, the interactions form a symmetric square matrix, $u_{i j}=u_{j i}$. Thus, the binding site of a protein is specified by giving values to the string of $n_{E}$ elements, and so there are $2^{n_{E}}$ possible different patches.

The binding constant $K_{i j}$ of a patch is given by [15]

$$
K_{i j}=(1 / 2)^{\delta_{i j}} \exp \left(u_{i j}\right) / 6,
$$

where the first factor is a symmetry factor which halves the equilibrium constant if $i=j$. The factor of $1 / 6$ is a normalisation factor of $1 / 24$, from the 24 distinct orientations, times the 4 possible rotations about the axis joining the centres of the proteins, all of which allow binding.

So, our interaction is mediated by patches, of which we have $N$ patches interacting in pairs. We neglect the other patches on the surface of the proteins, simply assuming that they are highly hydrophilic, having few hydrophobic elements, and so can be neglected. We do not need to specify the number of proteins $N_{P R}$, although of course as each protein has 6 patches, we must have that $N_{P R} \geq N / 6$.

Although we are focusing on proteins, a mixture of particles that satisfies equation (1) will selfassemble in a controlled way, the particles which have patch 1 will only stick to those that have patch 2 , those with patch 3 stick to those with patch 4 and so on. We can even consider macroscopic objects with interaction energies determined by sequences made of large hydrophobic and hydrophilic stripes, e.g., a cube just as in figure 1 and perhaps a few $\mathrm{mm}$ or $\mathrm{cm}$ across. If these objects in water are agitated sufficiently strongly to break the weak bonds, those with $K<K_{s}$, but not the stronger bonds, those with $K>K_{b}$, then the cubes should self assemble. For systems roughly equivalent to our model with small $n_{E}$, this has been done in a series of beautiful experiments by Whitesides and coworkers $[17,18]$.

\section{Calculation of the capability}

As each patch must recognise a unique other patch, each patch must have a unique sequence of hydrophobic and hydrophilic elements. As with $n_{E}$ elements there are $2^{n_{E}}$ different sequences then 
immediately we know that $2^{n_{E}} \geq N$, or $n_{E} \geq \log _{2}(N)$. This is an obvious lower bound on the number of elements required for proteins to bind specifically to 1 other protein from $N$. It does not take into account our requirement, equation (1). If we use all $2^{n_{E}}$ sequences then many sequences in the mixture will differ from other sequences present by the value of only 1 element. Yet we require that a patch on a protein bind very strongly to one other patch and very weakly to all the others. For example, we require that patch 1 bind to patch 2 strongly but patch 3 very weakly or not at all. Thus, we require that $K_{12}>K_{b}$ and $K_{13}<K_{s}$. But if say proteins 2 and 3 differ in only one element then $K_{12}$ and $K_{13}$ can differ by a factor of at most $\exp (\epsilon)$. So, then $K_{12} / K_{13} \leq \exp (\epsilon)$, which is inconsistent with our requirements for $K_{12}$ and $K_{13}$ unless $\epsilon$ is large. In other words as the ratio $K_{b} / K_{s}$ is large, in order satisfy our criterion for functionality, equation (1), we cannot have 2 sequences present in which only 1 element is different. This requirement to 'space out' the patches dramatically reduces the number of proteins we can simultaneously use in a mixture. We will need considerably more than $\log _{2}(N)$ elements per protein patch to achieve a functional mixture.

In fact this problem of a patch picking out one other patch from a total of $N$ patches is analogous to problems that arise in the study of communication through noisy channels. There the receiver of a message must pick out which of the possible messages they have received. The study of communication channels gave birth to what is now often called information theory [13]. In the 1940s Shannon wanted to know how many messages could be sent through a given noisy channel during some time interval, with each message being received correctly $[2,3]$. Reference [2] is reprinted in [3]. The theory is statistical in nature, in the sense that it does not consider a specific message but considers typical messages. Our theory too is statistical, we will not consider a specific mixture.

Now, we require that a protein bind to another with a large equilibrium constant $K_{b}$. This will require that the protein have a minimum of $B$ hydrophobic elements, where

$$
B=\operatorname{int}\left(\ln \left(6 K_{b}\right) / \epsilon\right)+1,
$$

from equation (3). The function int $(x)$ yields the largest integer less than $x$. It is required as the number of elements $B$ must of course be an integer. As we will see, the more hydrophobic elements a patch has the more other patches it sticks to, i.e., the more other possible patches have $K_{i j}>K_{s}$, and so the number of hydrophobic elements should be kept to a minimum. We demonstrate this at the end of this section. As it cannot be less than $B$ while still binding strongly to one other patch, the optimum number of hydrophobic elements is $B$. From now on, we only consider patches with $B$ hydrophobic elements. With this restriction the number of possible patches, $N_{P O S}$, is

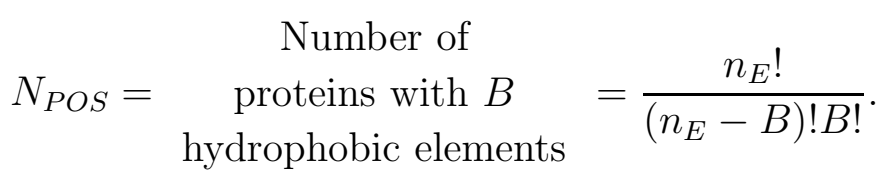

Actually, this neglects the fact that some of these sequences are not actually possible as they bind to themselves with $K_{i i}>K_{s}$, and this makes equation (5) a slight overestimate. The mean number of interactions of a patch with itself is close to $B^{2} /\left(2 n_{E}\right)$. In the next section we will be considering patches where $B^{2} /\left(2 n_{E}\right)$ is fewer than the number of hydrophobic interactions that make an interaction 
too sticky. So we will use equation (5) and neglect the fact that a small fraction of patches stick to themselves.

But any one of the possible proteins will stick to many other proteins. We use the term 'stick to' to mean bind with an equilibrium constant $K_{i j}>K_{s}$. We need to calculate the number of proteins $j$ for which $K_{i j}>K_{s}$. We denote this number by $N_{S}$. If $S$ is the minimum number of elements for which $K_{i j}>K_{s}$, then $S$ is given by

$$
S=\operatorname{int}\left(\ln \left(6 K_{s}\right) / \epsilon\right)+1 .
$$

Now, if $N_{s}$ is the number of patches with $B$ hydrophobic elements, $s$ of which interact with a fixed set of $B$ elements on another patch, then the number of patches that stick to a given patch, $N_{S}$, is

$$
N_{S}=\sum_{s=S}^{B} N_{s},
$$

because any patch with $s \geq S$ hydrophobic interactions contributes to the number of patches that are too sticky. Calculating $N_{s}$ is a simple exercise in combinatorics. Consider a patch with $B$ elements. The number of ways, $N_{s}$, that $s$ elements can be situated such that they interact with a fixed pattern of $B$ elements out of $n_{E}$ is

$$
N_{s}=\frac{B !}{(B-s) ! s !} \frac{\left(n_{E}-B\right) !}{\left(n_{E}-B-(B-s)\right) !(B-s) !} .
$$

Having found expressions for the total number of patches, $N_{P O S}$, and the number each one sticks to, $N_{S}$, we proceed to see how many specific interactions are possible. The first thing to note is that once a patch is specified, so is its partner. All $B$ of the partner's hydrophobic elements must be at the $B$ positions that interact with the hydrophobic elements of the first protein and so there is no freedom in choosing its sequence of elements. Thus, as there is a unique partner for each patch, we have $N_{P O S} / 2$ binding pairs of patches. Actually a very small fraction of patches bind to themselves, but we neglect this.

So, we have $N_{P O S} / 2$ pairs. Every time we add one of these pairs to the mixture, we have to discard approximately $2 N_{S}$ of these pairs. This figure of $2 N_{S}$ is obtained by making two assumptions. The first is that each of the 2 patches of the pair eliminates $N_{S}$ pairs by sticking to one of the partners we neglect sticking to more than one patch of a binding pair. The second is that there is no overlap between the sets of approximately $N_{S}$ pairs that each of the 2 patches of the pair added sticks to. If every time we add a pair we have $2 N_{S}$ fewer pairs then clearly we run out of pairs after we have added $\left(N_{P O S} / 2\right) /\left(2 N_{S}\right)$ pairs. We name the maximum number of patches that can bind together in pairs in a mixture, the capability and denote it by $C$. For our model it is

$$
C\left(n_{E}, \epsilon\right)=\frac{N_{P O S}}{2 N_{S}} .
$$

The maximum number of high specificity bonds is of course half the capability $C$. In information theory, the fundamental quantity is the capacity $C_{S h}$. In units of bits per bit sent, it is related to our capability by $C_{S h}=\log _{2}(C) / n_{E}[2,3,13]$. In communication the analogue of our capability $C$ increases exponentially so it is convenient to use not the capability itself but its logarithm the capacity. As we will see our $C$ 
does not increase exponentially with $n_{E}$ and so it is more convenient to work with it directly. In an appendix we discuss the similarities and differences to communication.

To derive equation (9) we assumed that $n$ patches stick to $n N_{S}$ others, neglecting any overlap between the $N_{S}$ patches that stick to one patch and the $N_{S}$ patches that stick to another. A simplifying assumption that means that equation (9) will tend to underestimate the true capability.

For their pioneering study of the immune system, Perelson and Oster [11] introduced the idea of 'shape space' of proteins where if a protein from the immune system is near in this space to a foreign protein, it will bind to, recognise, the foreign protein. Our model proteins too have a corresponding space and the capability we have calculated essentially corresponds to the number of proteins required to fill this space. By fill we mean have so many proteins that any extra one of the $N_{P O S}$ patches with $B$ hydrophobic elements that is added is likely to stick to one of the patches in the mixture. For an immune system to function the space should be overfilled to avoid any foreign protein escaping detection $[9,11,12]$, it is an advantage for more than one immune-system protein to bind to a foreign protein, whereas for specific binding the space must be underfilled.

When the first term in equation (7) for $N_{S}$ is the dominant one, we can approximate $N_{S}$ as

$$
\begin{aligned}
N_{S} & \simeq \frac{B !}{(B-S) ! S !} \frac{\left(n_{E}-B\right) !}{\left(n_{E}-B-(B-S)\right) !(B-S) !} \\
& \simeq \frac{B ! n_{E}^{B-S}}{S !(B-S) !},
\end{aligned}
$$

where in the second line we simplified the expression assuming $n_{E}-B$ is not too small. Making similar approximations for $N_{P O S}$ we get $N_{P O S} \simeq n_{E}^{B} / B$ ! and hence for the capability $C$,

$$
C \simeq \frac{1}{2} \frac{n_{E}^{B}}{B !} \frac{S !(B-S) !^{2}}{B ! n_{E}^{B-S}}=\frac{(B-S) !^{2} S !}{2 B !^{2}} n_{E}^{S} .
$$

Also, note that from this approximate expression it is clear that to maximise the capability the number of hydrophobic elements, $B$ in equation (11) should be as small as possible. In equation (11), the $B$ dependence is $C \sim 1 /[B(B-1) \ldots(B-S+1)]$ and this is a monotonically decreasing function of increasing $B$. Finally, as promised $C$ does not increase exponentially with the number of elements $n_{E}$. We should note that Shannon's channel-capacity theorem $[2,3]$ implies that there potentially exists a model in which $C$ does increase exponentially. So real proteins may have a $C$ that increases exponentially with the number of amino acids in a binding patch, however in our model the increase is sub-exponential.

\section{Results for the capability}

The binding strength of protein interactions are often given in terms of the dissociation constant, typically with Molar (M) units [10]. 1 Molar is $6 \times 10^{23}$ molecules per litre, which is 0.6 molecules $/ \mathrm{nm}^{3}$. The dissociation constant, $K_{d}$, for $A$ and $B$ binding together is, by definition, $K_{d}=c_{A} c_{B} / c_{A B}$, where $c_{A}, c_{B}$ and $c_{A B}$ are the Molar concentrations of free (unbound) $A$, free $B$ and bound $A B$ dimers, respectively [10]. It is therefore just one over the equilibrium constant for binding. Dissociation constants of protein interactions vary over orders of magnitude. They can easily be of order $\mu \mathrm{M}$ and in some cases values as low as $10^{-16} \mathrm{M}$ have been measured. For example, some E. coli can produce a protein called E9 (it is one of 
Figure 2. Plots of the capability $C$ as a function of the number of elements $n_{E}$. For both curves $\epsilon=1$ and the maximum permitted stickiness is $K_{s}=100(S=7)$. For the solid curve the binding strength is $K_{b}=10^{6}(B=16)$ and for the dashed curve $K_{b}=10^{8}(B=21)$. The dotted curves just below the solid and dashed curves are the results of the approximation equation (11) for $C$.

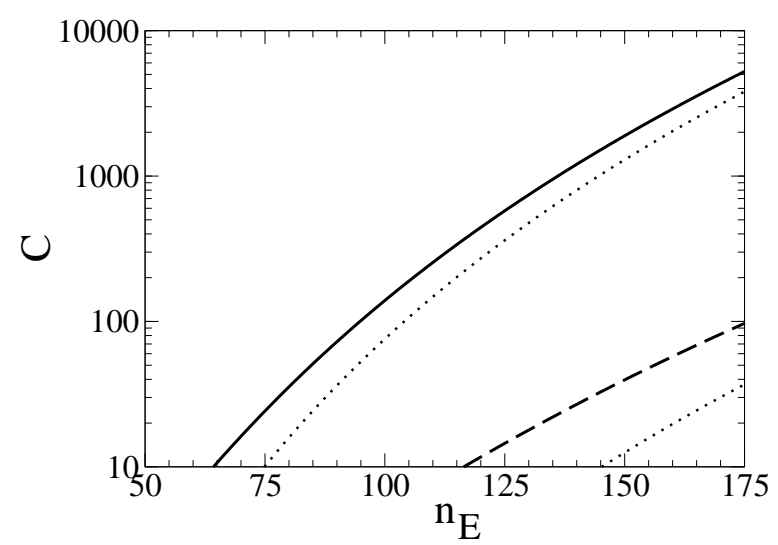

a family of related proteins) which is highly toxic, it is an antibiotic. E9 is neutralised by another protein, Im9, which binds to it to form a heterodimer [19-21]. At low salt in vitro the dissociation constant for the binding of Im9 to E9 is close to $10^{-16} \mathrm{M}$. In $250 \mathrm{mM}$ sodium chloride solution the dissociation constant is a mere $10^{-14} \mathrm{M}$ : the binding has a significant electrostatic component. This is a remarkably strong and specific binding, it must be specific as it must avoid binding in vivo to proteins other than E9.

E9 and Im9 are a somewhat extreme example, to get a feel for more typical values, consider a bacterium such as $E$. coli. It has a cytoplasm with a volume around $1 \mu \mathrm{m}^{3}$. So, one protein in this volume has a concentration of $10^{-9} \mathrm{~nm}^{-3}$ or about $1 \mathrm{nM}$. If there are only say 10 copies of $A$ and $B$ then for a significant fraction of these copies to bind to each other, then a dissociation constant around a $10 \mathrm{nM}$ is required. If there are around 1000 copies of the proteins $A$ and $B$ in each $E$. coli cell then a dissociation constant of around $1 \mu \mathrm{M}$ is enough to ensure roughly equal numbers of the free $A$ and $B$ and of $A$ and $B$ bound into dimers.

Our proteins are 2 lattice sites across and our equilibrium constants $K_{i j}$ are in units of the volume of a lattice site. Proteins are a few nms across and so each lattice site corresponds to a volume about 1 to $2 \mathrm{nms}$ across. Taking a lattice site to be $1 \mathrm{~nm}$ across, and noting that 1 Molar is close to $1 \mathrm{per}^{\mathrm{nm}} \mathrm{n}^{3}$ we have that $K_{i j}=1$ is approximately equivalent to an equilibrium constant of $1 \mathrm{M}^{-1}$. Thus to obtain a dissociation constant of $1 \mu \mathrm{M}$ we need a $K_{i j} \approx 10^{6}$, and so need to set $K_{b}=10^{6}$.

In figure 2 we have plotted the capability $C$ for $K_{b}=10^{6}$ (solid curve) and $10^{8}$ (dashed curve). In both cases we insisted that the equilibrium constant between patches not binding to each other be less than $K_{s}=100$. A $K_{b}=10^{8}$ is approximately equivalent to a dissociation constant of $10 \mathrm{nM}$. We took the energy of interaction between hydrophobic elements $\epsilon=1$. Then for $K_{b}=10^{6}, B=16$ and, for $K_{b}=10^{8}, B=21$, and with $K_{s}=100, S=7$ elements is too sticky, $K_{i j}>100$. Also, the dotted curves are the result of equation (11). 
Figure 3. Plots of the number of possible proteins to which a protein binds, $N_{S}$, as a function of the number of elements $n_{E}$. For both curves $\epsilon=1$ and the maximum permitted stickiness is $K_{s}=100$ $(S=7)$. For the solid curve the binding strength is $K_{B}=10^{6}(B=16)$ and for the dashed curve $K_{B}=10^{8}(B=21)$.

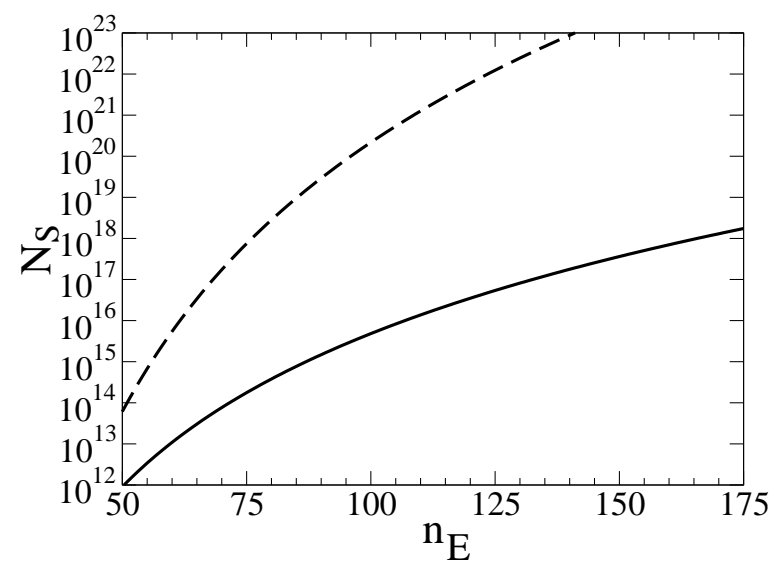

Of course the capability $C$ increases as $n_{E}$ increases but for the stronger binding, even for $n_{E}=150$, $C=40$. This is highly inefficient, the restriction to be functional means that we need 150 elements for a mixture with 40 specific binding interactions, whereas if we just needed 40 different patches then $\log _{2} 40=5.3$ elements would have been enough. However, each patch of a protein is required to bind to 1 other patch with a very large equilibrium constant, and with equilibrium constants at least 6 orders of magnitude smaller to any of the other 39 patches. This requirement is a very stringent one.

The inefficiency can be clearly seen in figure 3 where we have plotted the number of possible patches to which a patch sticks. We see that if we insist on specific binding of strength $K_{b}=10^{8}$ (the dashed curve) then we require $B=21$ hydrophobic elements for bonding of this strength but then when $n_{E}=150$, a patch with 21 hydrophobic elements sticks to approximately $2.8 \times 10^{23}$ other possible proteins. The equilibrium constant between a single patch and of order $10^{23}$ others is greater than $K_{s}=100$. Thus to make a mixture that is functional according to our criteria, for every specific-binding patch we add to the mixture the number of possible patches that we can no longer add to the mixture increases by $10^{23}$.

The same thing must apply to real proteins in vivo, although proteins are much more complex than our, deliberately very simple, model and so it is not possible to quantify $N_{S}$ for real proteins. However, we can return to our example of E9 and Im9 [19-21]. A number of single-residue mutants have been made for approximately 30 of the residues of Im9 suspected of contributing to the E9-Im9 binding. The changes in the dissociation constant in each case were measured, and they cover a large range [20] but many of them resulted in only modest increases in the dissociation constant. Thus, many sequences with these mutations are not viable as part of the amino-acid sequences of proteins. We cannot have, for example, an enzyme that has a part of its amino-acid sequence close to that of Im9 as the enzyme will 
bind to E9. Many more mutants, mutants with 2, 3, etc. mutations, will also bind strongly to E9 and in general this will be inappropriate. So functional proteins cannot include these amino-acid sequences, thus reducing the number of amino-acid sequences which are functional in the mixture of proteins in the cytoplasm of E. coli when E9 is present.

\section{Conclusion}

The mixture of proteins inside cells is not simply an inert mixture like, for example, the complex mixture of hydrocarbons in crude oil. It has evolved to be functional: the proteins do things. In particular, many proteins must bind strongly to a specific patch or patches on one or more other proteins. Here, we studied a very simple model protein, introduced in [15]. We borrowed ideas from the theory of communication, and calculated the maximum number of specific binding interactions that can function together in a mixture. We called it the capability $C$. It is roughly the number of possible different patches, $N_{P O S}$, over the number of patches that stick to a given patch and so cannot be in the same mixture, $N_{S}$, $C=N_{P O S} / 2 N_{S}$, The capability $C$ of the constituent proteins limits the size of the interactome. The interactome is a network or web of many specific interactions, and it can have at most $C / 2$ links. A network with many links requires that the proteins of which it is composed must have large patches, patches with a large number of elements.

Schneider has applied information theory to analysing the DNA sequences of sequences of base pairs to which proteins bind with high specificity $[4,6]$. We briefly apply an analogous analysis to our model in the appendix. Work on the size of the regions on foreign proteins that are recognised by the immunesystem proteins is very similar in approach to that used here [9]. For example, Percus et al.'s [12] study of how demanding is the requirement that our immune-system proteins differentiate between foreign and human proteins, is analogous to our study of how demanding is the requirement that a protein bind to only one other patch in a mixture. However, we do use a concrete although highly simplified, model of a protein unlike work on the immune system which has used rather abstract models of recognition. Indeed, it might be useful to use our concrete model of a protein in future studies of the immune system.

The more specific the binding, the larger $K_{b} / K_{s}$ at fixed $K_{s}$, the more elements $n_{E}$ we need to achieve a given capability $C$, see figure 2. Now, although there is no one-to-one correspondence between our hydrophobic/hydrophilic elements and the residues of a real protein, we expect that increasing $n_{E}$ corresponds in real proteins to increasing the size of the protein. But ideally, proteins should be as small as possible, they will then consume less material (amino acids), diffuse faster, and take up less volume. For these reasons we might want $n_{E}$ to be as small as possible and so there is a trade off between the size and complexity needed for specific binding and the problems of being large. This trade off involves not only the properties of an individual protein but also the global properties of the mixture, e.g., the number of components. Eukaryote cells are compartmentalised [10] and each compartment will have only a subset of the total number of proteins. A smaller number of proteins with higher concentrations in a compartment allows strong specific binding at smaller values of $n_{E}$.

Very recent work on the specificity of protein-protein binding has been done by Havranek and Harbury [22], and by Shifman and Mayo [23]. They looked at specificity but in both cases they used 
a combination of a much more detailed model and experiments. In particular, Havranek and Harbury considered 2 proteins and constrained each protein to: i) to dimerise with itself, ii) not to bind to the other protein, iii) not to aggregate. They found that proteins have to be specifically engineered not to bind to other proteins and not to aggregate, as well as to bind to their binding partner (themself in this case). The detailed nature of their model restricted them to a mixture of 2 proteins. It also precluded the exhaustive enumeration of all possible proteins and elimination of all of the proteins that bind to proteins other than their partners, that we performed here. Following the approach taken by Shannon $[2,3]$ we also did not consider a specific mixture, as Havranek and Harbury did, but calculated a limit on what the mixture could do, here the limit on the number of interaction of a given specificity. However, theirs and our approaches are related. We both consider how specificity is achieved and in doing so explicitly consider the binding of a protein to the wrong other protein or site, that must be prevented. This avoidance of an undesired state is referred to as negative design [24-26].

Future work could also go in a number of other directions. The concepts introduced here of a capability $C$ for specific binding of a protein could be applied to other models of proteins. Also, the proteins in a cell are the product of evolution of course, and it would be interesting to study the constraints on evolution of proteins due to the requirement of specific binding. Very recent work [27-29] on large data sets of interactions, partial interactomes, has found a negative correlation between the rate of evolution of the amino-acid sequence of a protein and the number of specific interactions it participates in. Although there is some debate over this finding [29-31]. This work relies on recent work characterising very large numbers of interactions, particularly in yeast [32,33]. Also see the references of $[27,28]$ for earlier work on individual proteins. Within our model, if a protein patch is part of a mixture near the capability of its proteins, then not only would many mutations be deleterious because they weakened its binding to its partner, many mutations would be deleterious because they caused the patch to stick to other patches in the mixture. The mutation rate of an individual protein will in general depend on the sequences of elements of all the other patches, not just that of its partner.

It is a pleasure to acknowledge that this work started with inspiring discussions with D. Frenkel. I would also like to acknowledge discussions with J. Cuesta, and J. Doye and A. Louis for introducing me to negative design. Finally, I would like to thank an anonymous reviewer for drawing my attention to the connection to the work of Whitesides. This work was supported by the Wellcome Trust (069242).

\section{Appendix}

In our notation, the channel-capacity theorem of Shannon states that in the $n_{E} \rightarrow \infty$ limit, we can achieve a capability $C$ such that $\log _{2}(C) / n_{E} \rightarrow O(1)$, with fixed $K_{s}$ and the specificity ratio $K_{b} / K_{s}$ arbitrarily large. For our model protein $C$ does not increase exponentially with $n_{E}$ and so in the $n_{E} \rightarrow \infty$ limit, $\log _{2}(C) / n_{E} \rightarrow 0$. Thus the channel-capacity theorem does not apply to our model of a protein. Note that this does not rule out a different type of model protein for which the channel capacity theorem holds, or that real proteins might obey it.

Shannon's derivation of the channel-capacity theorem (theorem 11 of $[2,3]$ ) breaks down when

applied to our proteins because he assumes, correctly for communication, that the entropy per bit sent 
of the strings is some constant which he calls $H$. Here the entropy per element $s$, is

$$
s=-\left(\frac{B}{n_{E}}\right) \ln \left(\frac{B}{n_{E}}\right)-\left(\frac{n_{E}-B}{n_{E}}\right) \ln \left(\frac{n_{E}-B}{n_{E}}\right)
$$

which is the logarithm of equation (5) over $n_{E}$. At fixed $\epsilon, B$ is fixed and so as $n_{E}$ is increased, $s$ decreases. In order to avoid the patches becoming very sticky the number of hydrophobic elements must be minimised and this makes $s$ decrease with increasing $n_{E}$ which in turn dramatically reduces the capability. This feature is present in our model but not in communication systems. It is also not present in the more abstract approach to applying information theory to high specificity binding of Schneider $[4,7]$. It is possible that it is simply an artifact of our simple model, but other related models do show a similar behaviour [34]. Another perspective on this is to note that to make $s$ not a function of $n_{E}, B$ should not be a constant, it should be $f n_{E}$, with $f<1$ a constant. But then the average interaction energy between 2 patches is $\epsilon f^{2} n_{E} / 2$ : which increases with $n_{E}$. In essence the problem is that the larger the number of elements of the patch the larger the interaction energy is. It is possible that a similar problem also occurs in real proteins, where if a large patch on the surface of a protein is required to achieve binding of high affinity it is difficult to avoid this large patch interacting strongly with many other proteins. The analogue of the $\log \left(N_{S}\right) / n_{E}$ in communication, called the equivocation, is also a constant, unlike $\log \left(N_{S}\right) / n_{E}$.

Finally, it should be noted that $s n_{E}$ is simply the entropy associated with the space of proteins that have the right number of hydrophobic elements. This is the space consisting of $N_{P O S}$ proteins. It is not an information in the sense that the information of binding sites on DNA evaluated by Schneider $[4,6]$ is an information. He evaluates the information about a DNA sequence that you obtain if you are told that the sequence is the binding site for say the protein LacI [6]. This information, call it $I_{s}$, is, by definition, the difference between the entropy of the DNA sequence $(2 \times$ number of base pairs if the 4 base pairs all occur with probability 0.25 ) if you know nothing about it and the entropy of sequences that LacI binds too. We can evaluate a similar information, again call it $I_{s}$, for our model proteins. Let us consider protein $i$, with $i$ odd so that it binds to protein $i+1$. $I_{s}$ is the information about the sequence of protein $i$ that you obtain if you are told what the sequence is of protein $i+1$. In section 4 when we calculated the capability $C$ we insisted on proteins having $B$ hydrophobic elements and then once the sequence of protein $i+1$ is fixed, so is that of protein $i$ and so the entropy of sequence $i$ is then 0 once the sequence of $i+1$ is known. Then $I_{s}=s n_{E}$. But $I_{s}$ need not always equal $s n_{E}$. In a mixture of proteins with interactions coded by $n_{E}$ elements, with $N$ below $C\left(n_{E}\right)$ more than one sequence $i$ will bind to $i+1$ and also satisfy the requirement not to stick to the other $N-1$ proteins. The number of hydrophobic elements will not always be $B$. Then the entropy of protein $i$ once the sequence of protein $i+1$ has been specified will be greater than 0 and $I_{s}$ will not equal the entropy of the space of possible proteins.

\section{References}

[1] Koundrioukoff S, Jónsson Z O, Hasan S, de Jong R N, van der Vliet P C, Hottiger M O and Höbscher U 2000 J. Biol. Chem. 27522822

[2] Shannon C E 1948 Bell System Technical J. 27 379; ibid 27, 623 
[3] Shannon C E and Weaver W 1949 The Mathematical Theory of Communication (University of Illinois Press, Urbana). This reference includes a reprint of [2].

[4] Schneider T D 1994 Nanotechnology $\mathbf{5} 1$

[5] Schneider T D 2001 Nuc. Acids Res. 294881

[6] Schneider T D, Stormo G D, Gold L and Ehrenfeuch A 1986 J. Mol. Biol 188415

[7] Schneider T D 1991 J. Theo. Biol. 14883

[8] Gerland U, Moroz J D and Hwa T 2002 Proc. Nat. Acad. Sci. 9912015

[9] Perelson A S and Weisbuch G 1997 Rev. Mod. Phys. 691219

[10] Alberts B, Bray D, Lewis J, Raff M, Roberts K and Watson J D 1994 Molecular Biology Of The Cell (3rd Edition, Garland Publishing, New York).

[11] Perelson A S and Oster G F 1979 J. Theo. Biol. 81645

[12] Percus J K, Percus O and Perelson A S 1993 Proc. Nat. Acad. Sci. 901691

[13] MacKay D J C 2003 Information Theory, Inference, and Learning Algorithms (Cambridge University Press, Cambridge)

[14] Colas P, Cohen B, Jessen T, Grishna I, McCoy J and Brent R 1996 Nature 380548

[15] Sear R P 2003 J. Chem. Phys. in press; cond-mat/0306440

[16] Sear R P 2002 Europhys. Lett. 60938

[17] Whitesides G M and Grzybowski B 2002 Science 2952418

[18] Bowden N, Terfort A, Carbeck J and Whitesides G M 1997 Science 276233

[19] Wallis R, Moore G R, James R and Kleanthous C 1995 Biochem. 3413743

[20] Wallis R, Leung K-Y, Osborne M J, James R, Moore G R and Kleanthous C 1998 Biochem. 37476

[21] Kuhlmann U C, Pommer A J, Moore G R, James R, Kleanthous C 2000 J. Mol. Biol. 3011163

[22] Havranek J J and Harbury P B 2003 Nature Struct. Biol. 1045

[23] Shifman J M and Mayo S L 2003 Proc. Nat. Acad. Sci. 10013274

[24] Richardson J S and Richardson D C 2002 Proc. Nat. Acad. Sci. 992754

[25] Wang W and Hecht M H 2002 Proc. Nat. Acad. Sci. 992760

[26] Doye J P K, Louis A A and Vendruscolo M, preprint.

[27] Fraser H B, Hirsh A E, Steinmetz L M, Scharfe C and Feldman M W 2002 Science 296750

[28] Teichmann S A 2002 J. Mol. Biol. 324399

[29] Fraser H B, Wall D P and Hirsh A E 2003 BMC Evo. Biol. 311

[30] Jordan I K, Wolf Y I and Koonin E V 2003 BMC Evo. Biol. 31

[31] Bloom J D and Adami C 2003 BMC Evo. Biol. 321

[32] Uetz P et al 2000 Nature 403623

[33] Ito T et al 2000 Proc. Nat. Acad. Sci. 971143

[34] Sear R P unpublished work. 